\title{
Biography of biophenols: past, present and future
}

\section{Hassan K. Obied}

Graham Centre for Agricultural Innovation, School of Biomedical Sciences, Charles Sturt University, Wagga Wagga, NSW, 2678 Australia

Corresponding Author: Hassan Obied, PhD, Graham Centre for Agricultural Innovation, School of Biomedical Sciences, Charles Sturt University, Wagga Wagga, NSW, 2678 Australia

Submission date: April 28, 2013; Acceptance date: June 1, 2013; Publication date: June 24, 2013

\begin{abstract}
:
Background: The term "biophenols" was first coined by Romeo and Uccella in 1996 to denote bioactive phenols in olives replacing the more common and less chemically accurate term "polyphenols". "Biophenols" has started gaining popularity beyond olive chemistry and currently used by researchers to refer to plant phenols in general. Biophenols constitute the largest group of secondary plant metabolites with ubiquitous presence in plants and wide spectrum of biological activities. During the last three decades, biophenols have seized scientific attention, lured industry and attracted consumers' interest due to their antioxidative potential in preservation of food and maintaining human health. For the first time, a definition for "biophenols" has been proposed to present a more precise and comprehensive umbrella term to encompass all phenolic compounds of botanical origin. In this article, the author is tracing the landmarks of the history of biophenol research; profiling contemporary research directions; highlighting research controversies and uncertainties; and presenting an outlook of the biophenols future research.
\end{abstract}

Keywords: polyphenols, chemistry, pharmacology, biological activities, antioxidant

\section{BACKGROUND:}

The ability of food to maintain health and prevent disease is a common belief and a proven scientific fact. However, the power of food constituents to treat and cure ailments is a contested concept. The idea of using food for medication is not novel. Hippocrates famous quote, said centuries ago, "Let thy food be thy medicine and medicine be thy food" is marking food research during the last two decades. Research has been fuelled by a genuine interest from industry and consumers. Research shows health and nutritional claims have become a significant contributor to the customer's brand choice [1, 2]. The therapeutic potential of food is one of the hottest research topics. New research terms and disciplines have emerged; such as "nutrapharmacology", "medical food", "nutrigenomics", "nutriproteomics", "nutraceuticals", "medical nutrition", "functional food" etc. 
There is a multitude of entangled factors behind the surge in research examining medicinal properties of food. As the aging population is increasing steadily, there is more demand for healthier aging. The stressful life style and increased consumption of junk food have turned obesity into a worldwide epidemic. Overweight and obesity are major predisposing factors for chronic diseases such as cardiovascular diseases, diabetes, neurodegenerative diseases, and cancer [3]. Concomitantly, chronic diseases are the leading cause of mortality worldwide [4]. The magic bullet approach of orthodox Western medicine has repeatedly failed to cure or stop the progression of these complex diseases. Moreover, the adverse reactions of current drugs are frequently more serious than the disease itself. The situation for microbial diseases is not any better, with rising multi-drug resistant superbugs and a continuous decrease in the number of novel antimicrobials. Consequently, the need for more effective, safer and smarter alternatives has turned us back to dig for Mother Nature's treasures. No doubt, the most precious treasure of all is the green pharmacy plants.

The plant kingdom has kept offering remedies for humans from time immemorial. Nearly a quarter of our current drug arsenal is from higher plants [5]. Plants are still a major source for discovery of new lead compounds for the pharmaceutical industry. During the last two decades nearly $50 \%$ of the newly introduced drugs are of plant origin or analogues thereof [6]. Bioprospecting has been the classical pharmaceutical view of plants as sources for discovery of new lead compounds or potent bioactive molecules. As potency is a rare trait of phytochemicals [7], bioprospecting is gradually overridden by the more holistic and integral view of using plant mixtures, extracts or partially purified extracts. Researchers have started discovering traditional and ethnomedicine anew [8]. By 2016, the global nutraceutical market will exceed 207 billion U.S. dollars [9].

The most widely celebrated healthy diet is certainly the "Mediterranean diet" backed by a spate of epidemiological studies [10]. The early observations of low incidence of coronary heart disease CHD in Naples in 1952 by Ancel Keys and his later studies [11] laid the first cornerstone in the Mediterranean diet and health paradigm. The power of the Mediterranean diet to combat diseases and promote health was supported by the ground breaking findings of Serge Renaud in 1992 who coined the French Paradox [12]. While French diet is relatively higher in saturated fat, the incidence of CHD amongst French people was low. Though these findings have been a matter of huge controversy and were questioned in numerous studies. Wittingly or unwittingly, Keys and Renaud's findings have provided a significant impetus to study the active constituents of the Mediterranean diet. The common dominator among the main components of Mediterranean diet-whole grain cereals, legumes, vegetables, fruits, extra virgin olive oil and red wine- is a high content of plant phenolic compounds referred to as polyphenols or biophenols [13].

Biophenols are highly prized for their free radical scavenging and antioxidant activities. Oxidative stress happens when there is a serious imbalance between biological system ability to generate and detoxify reactive oxygen species (ROS). Oxidative stress is alleged to play a pivotal role in the aging process and in the pathology of age-related diseases. Hence, biophenols are co-opted to serve as natural antioxidants to alleviate or correct this oxidative imbalance. In many experimental studies, biophenols have demonstrated a wide spectrum of pharmacological activities beyond their antioxidant properties. The aim of the current review is to portray the status quo of biophenol research and to highlight important controversies and uncertainties in order to guide future research. 
What are Biophenols?: Biophenols constitute the largest group of secondary plant metabolites with ubiquitous presence in plants. Ironically, there is not a biochemically accurate and comprehensive term to describe plant phenols. The term "polyphenols" is the most frequently used term to describe plant phenolic compounds. It retrieves more than 79,000 hits in Google Scholar; 26,000 hits in Scopus; and 20,000 hits in ISI Web of Knowledge (April 2013). Originally, the term "polyphenols" was used to describe plant phenolic polymers, tannins [14]. The misuse of the term polyphenol to describe monomeric compounds is most likely inherited from wine chemistry. Robards and his co-workers repeatedly questioned the validity of using "polyphenol" to describe simple phenolic compounds with one aromatic benzene ring [15-17]. Quideau et al. have thoroughly investigated the history of polyphenol and critically scrutinized the use of the term "polyphenols" [18]. They redefined polyphenols as "plant secondary metabolites derived exclusively from the shikimate-derived phenylpropanoid and/or the polyketide pathway(s), featuring more than one phenolic ring and being devoid of any nitrogen-based functional group in their most basic structural expression" [18]. This has allowed the inclusion of plant phenols which have two phenolic rings such as flavonoids, depsides and phenolic oligomers. However, compounds with one phenolic ring are still not included. In addition, the nitrogen containing choline conjugates of phenolic compounds are excluded.

The term "biophenols" was first introduced by Romeo and Uccella in 1996 to describe bioactive phenols in olives [19]. Robards and co-workers [15] heralded the use of the term "biophenols" to refer to plant phenols in general as a more chemically accurate substitute of the complacent term "polyphenols" [18]. The term "biophenols" lends itself to be a more comprehensive and chemically accurate expression to describe plant phenols from simple monophenols to complex polymeric molecules. The prefix "Bio" denotes the biological origin as opposed to synthetic compounds. One important objection for the use of this term can be the multitude of phenolic compounds that are not naturally present in plant tissues but are produced as artefacts during post-harvest processing or handling of plant tissues: such as canolol in canola oil or isochromans and lignans in virgin olive oil. Hence, biophenols should be defined as "Phenolic compounds isolated from plant tissues or products that are derived from shikimate-phenylpropanoid and/or polyketide pathway(s) including their derivatives, conjugates, degradation products and metabolites". Thus, "biophenols" become an umberlla term that refers to all phenolic molecules derived from botanical origin.

Biophenol Research Timeline: It all started with the pioneer work of Denham Harman in the 1950's, the father of the Free Radical Theory of Aging. Harman demonstrated that dietary antioxidants can prolong the lifespan of terminally ill mice [20]. In 1960's, Sanojki proposed that degenerative diseases can result from overproduction of ROS [21]. It was not until 1972 that Harman denoted the mitochondrial role in the production of free radicals and the biological aging [22]. In 1985, Sies introduced the most seminal title on "oxidative stress" [23]. The 1980's were a turning point in the history of oxidative sress and antioxidants. The number of citations for the term "antioxidant" increased five folds while the term "oxidative stress" increased by more than 28 folds (Table 1). Concomitantly, the increasing safety concerns of synthetic antioxidants and the rise of naturotherapy ushered the hunt for natural antioxidants in 1990's. A 45-fold rise in "oxidative stress" publications and a ten-fold increase in manuscripts targeting polyphenols along with the emergence of the term "biophenols" are the main landmarks for biophenol research in the 1990's. During last 
decade, Table 1 shows a parallel 5-fold increase in all terms related to biophenol and antioxidant research. Biophenol research continued to prosper up with an average of 3000 manuscripts per year. The ardor of biophenol research is expected to exponentially increase during the current decade. An average of 6,500 research articles per annum have been published in 2011 and 2012 according to ISI Web of Knowledge.

Table 1. Number of publications in English according to the ISI web of Knowledge ${ }^{*}$

\begin{tabular}{|c|c|c|c|c|c|c|}
\cline { 2 - 7 } \multicolumn{1}{c|}{} & \multicolumn{5}{c|}{ Number of Publications } \\
\hline Years & Polyphenols & Biophenols & Antioxidants & $\begin{array}{c}\text { Free } \\
\text { radicals }\end{array}$ & ROS & $\begin{array}{c}\text { Oxidative } \\
\text { Stress }\end{array}$ \\
\hline $\begin{array}{c}1900- \\
1950\end{array}$ & 37 & 0 & 122 & 296 & 1 & 0 \\
\hline $\begin{array}{c}1951- \\
1960\end{array}$ & 107 & 0 & 321 & 736 & 0 & 1 \\
\hline $\begin{array}{c}1961- \\
1970\end{array}$ & 253 & 0 & 726 & 2,556 & 0 & 3 \\
\hline $\begin{array}{c}1971- \\
1980\end{array}$ & 452 & 0 & 1,738 & 5,357 & 51 & 31 \\
\hline $\begin{array}{c}1981- \\
1990\end{array}$ & 613 & 0 & 7,279 & 15,234 & 940 & 887 \\
\hline $\begin{array}{c}1991- \\
2000\end{array}$ & 6,244 & 17 & 65,182 & 76,364 & 25,159 & 39,759 \\
\hline $\begin{array}{c}2001- \\
2010\end{array}$ & 35,211 & 90 & 223,791 & 133,768 & 115,504 & 248,185 \\
\hline
\end{tabular}

* searching some common keywords such as "plant", "animal" and "human" showed that the general pattern is a doubling of the number of publications per decade.

Biophenols Research: Present: While alkaloids seized the scientific attention in the $20^{\text {th }}$ century and provided many drugs to the pharmaceutical industry, the $21^{\text {st }}$ century is going to witness the golden era of biophenol research (Figure 1). Flavonoids have been the most studied class of biophenols (Figure 1 and Figure 2). Amongst flavonoids, anthocyanins have captured most of the published research between 2000 and 2012, followed by flavones, flavonols and isoflavones (Figure 3). The most studied biophenol ever is the flavonol, quercetin. Catechins are next after quercetin. Concomitantly, the most studied flavonoid glucoside is quercetin-3-O-rutinoside commonly referred to as rutin. Resveratrol is the most investigated stilbene. Caffeic acid and its quinic acid conjugate, chlorogenic acid, are the most studied phenolic acids. Though biophenols are universal in plant kingdom, certain plants have been extensively studied for their biophenol content and advanced our understanding of biophenol chemistry and pharmacology. The blockbusters are tea, grapes (wine), apple, olives (virgin olive oil), berries, pulses, coffee and citrus fruits. 


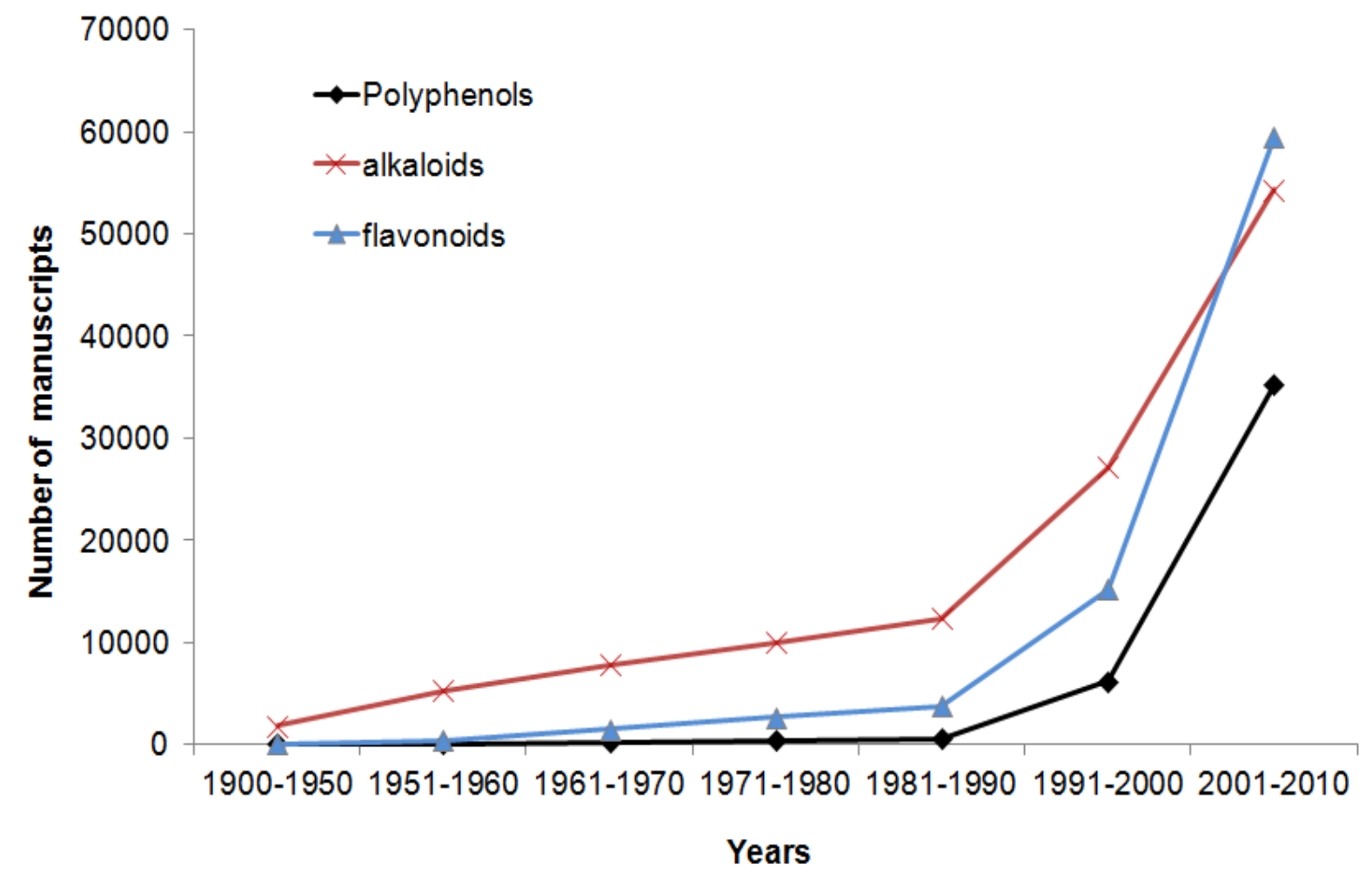

Figure 1. Number of manuscripts published on polyphenols, alkaloids and flavonoids as found in ISI Web of Knowledge database.

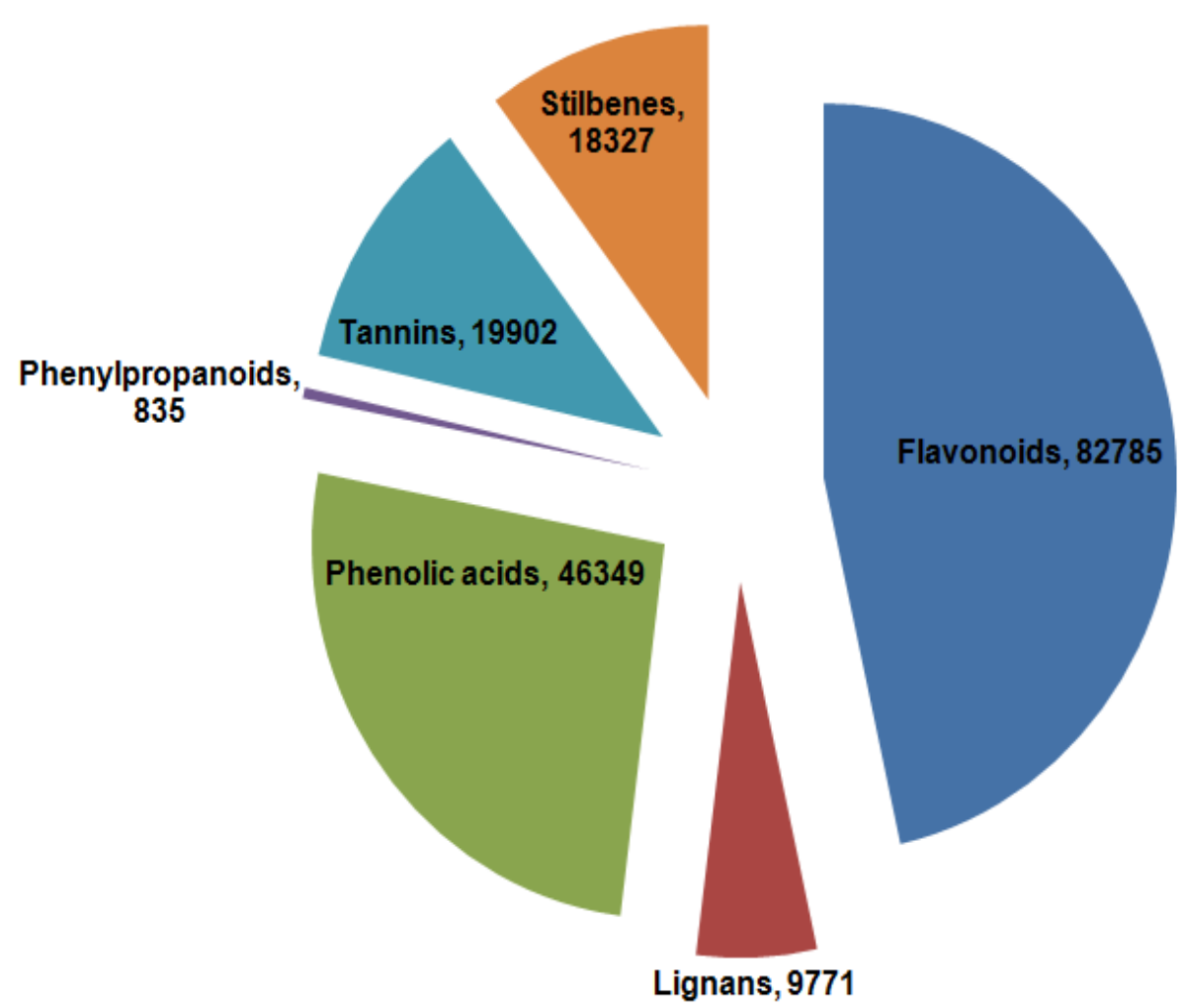

Figure 2. Survey of the number of published research articles on major biophenolic chemical classes in ISI web of Knowledge database 2000-2012. 


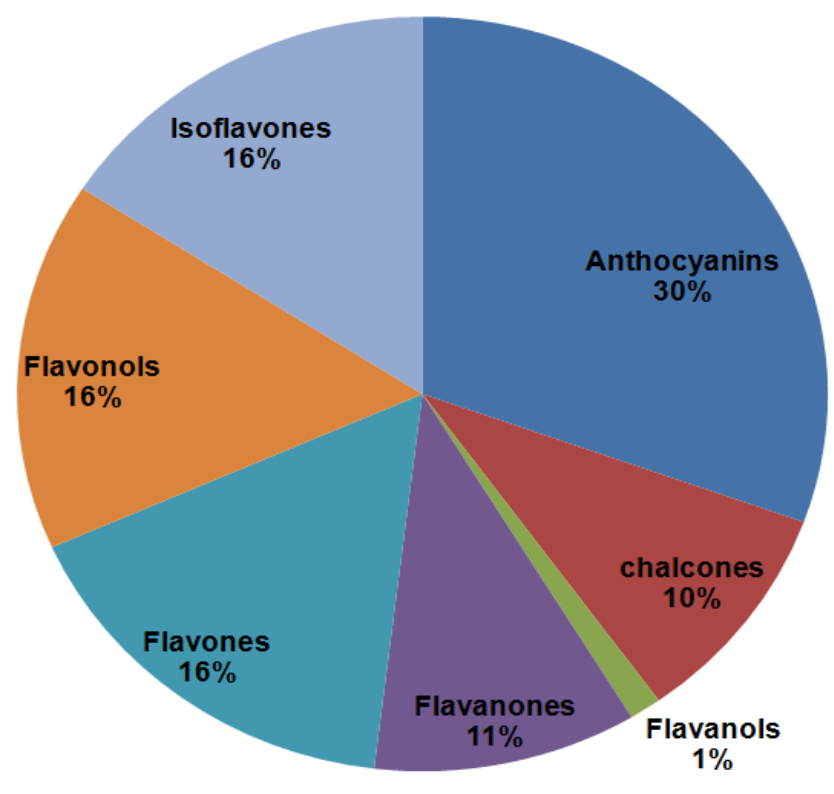

Figure 3. Survey of the most studied flavonoid classes as per number of publications in ISI Web of Knowledge database 2000-2012.

The growth of biophenol research is expanding horizontally as well as vertically. The scope of biophenol research overstretches from their origin in plant cells through their journey to the supermarket or pharmacy shelf and to their targets in human cells and up to their metabolism and renal/hepatic excretion. Figure 5 shows the key areas of contemporary biophenol research. Allured by commercial interest and scientific curiosity, researchers across a myriad of disciplines have rolled up their sleeves to explore the biophenol terrains and crack down the conundrum of biophenols in health and disease. The ubiquitous presence of biophenols -especially in traditional foods and herbal remedies- and the relatively inexpensive laboratory techniques generally employed in their research allowed scientists from developing as well as developed countries to take part (Figure 4).

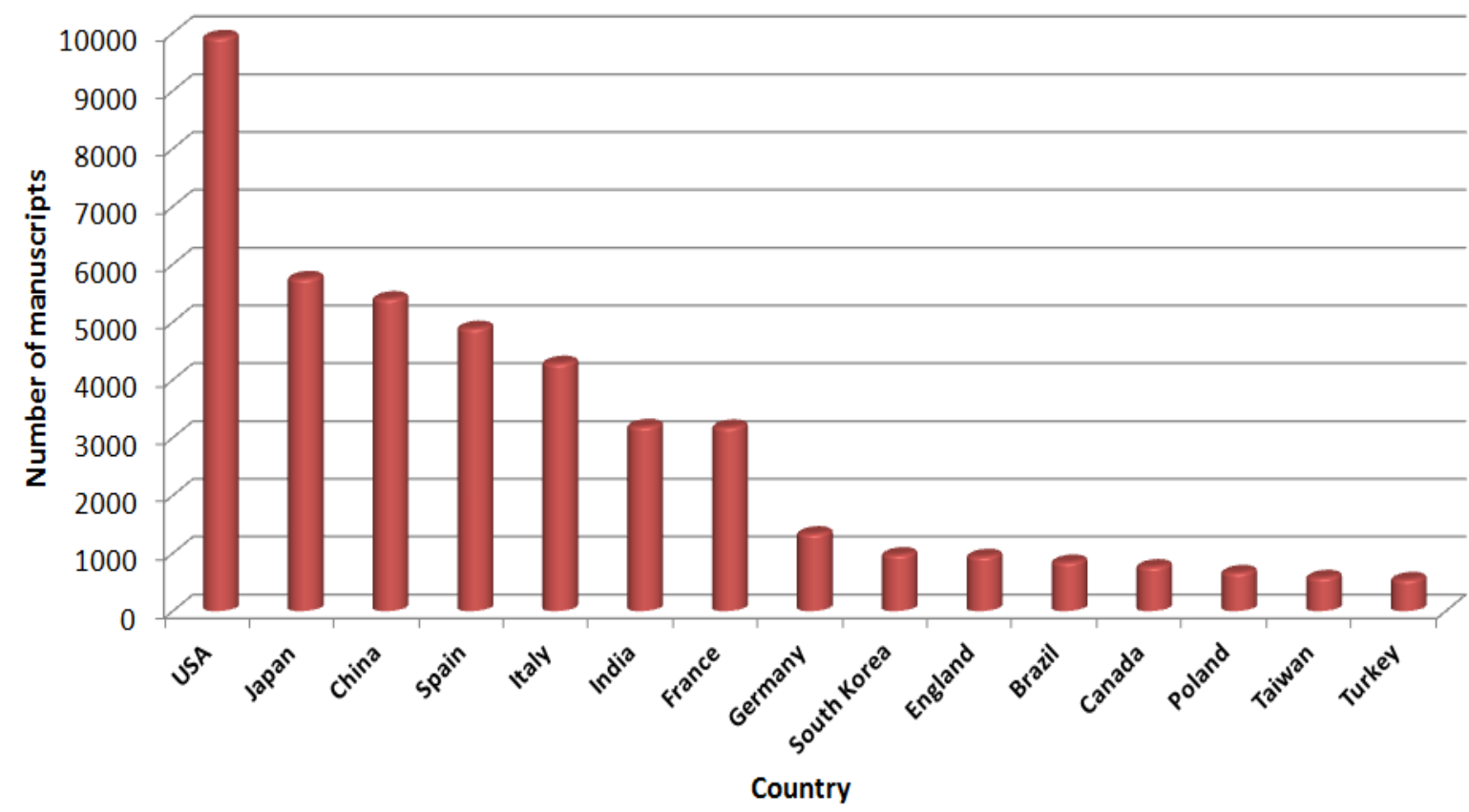

Figure 4. Top 15 countries in biophenol research output (published research manuscripts between 1900 and 2013) ISI Web of Knowledge database 


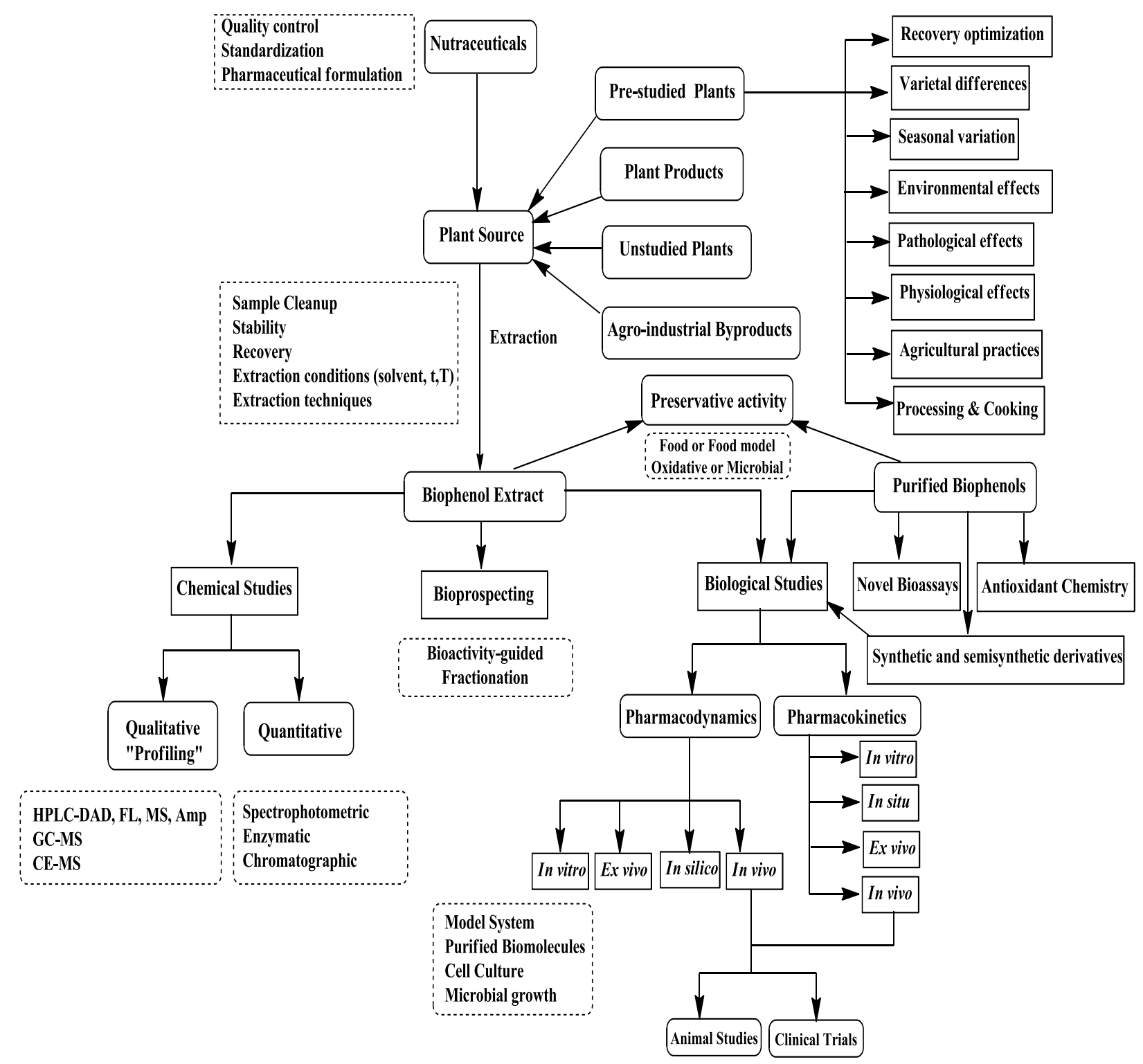

Figure 4. Key areas of biophenol research

What is the consensus?: Though researchers have demonstrated a broad spectrum of pharmacological and medicinal properties for biophenols, antioxidant activity is so far the central dogma. Biophenols show superior antioxidant potential in vitro. They effectively scavenge a multitude of non-biological free radicals, superoxide radical, hydrogen peroxide and various reactive oxygen, nitrogen and chlorine species [13, 24]. In addition, biophenols can exert antioxidant effects acting as reducing agents and metal chelators [13]. Antioxidant activity increases with the increase of the number of hydroxyl groups and the extent of conjugation of double bonds [25]. In cell lines and animal models, biophenols could upregulate endogenous antioxidant enzymes such as catalase, superoxide dismutase, quinine reductase, glutathione peroxidase, glutathione $S$-transferase and $\gamma$-glutamylcysteine synthetase [13]. Biophenols can also inhibit the prooxidant enzymes such as xanthine oxidase [26]. An increase in uric acid level in blood is suspected by some scientists to be the actual contributor to the antioxidant activity of biophenols in vivo [26]. On the other hand, biophenols may act as prooxidants in model systems and ex vivo, generally at higher concentrations required for antioxidant activity [13, 27]. There is no clinical data on 
prooxidant activity and equivocal data on antioxidant activity of biophenols [28, 29]. Most of the studies conducted used plant extracts or biophenol-rich food rather than pure single agents. Generally, the maximum plasma concentrations of biophenols are not enough to produce considerable antioxidant effects [29]. Accumulating evidence suggests that biophenols have good oral bioavailability yet the low plasma concentrations are due to extensive first pass metabolism [13]. Nevertheless, several clinical trials report significant reduction in some oxidative and pathological markers [13, 30]. Hitherto the consensus is consumption of diet rich in fruits and vegetables is linked to resistance to age-related diseases, while the biophenol-antioxidant role is still inconclusive.

Pharmacological activities of biophenols are so diverse and intricate. Biophenols are reported to affect various body systems, modulate immunity and fight pathogens and neoplasms [13]. Further, biophenols are proclaimed to affect mood, behaviour, memory and cognition [31-35]. Biophenols demonstrated- at least in vitro- abilities to modulate cell signalling pathways, react with specific receptors, interfere with cell division, modulate enzyme activities, regulate gene expression and affect epigenetics [13].

What is controversial?: Indeed, the biggest consensus about biophenol research is its controversy. Biophenol definition, function in plant, function in humans and mechanisms of action are all contentious issues. For long, biophenols were taunted as anti-nutrients as they bind to proteins and handicap their bioavailability [36]. Currently, biophenols are categorized as important micronutrients [37]. One of the most interesting controversies is whether biophenols are antioxidants or prooxidants. Professor Barry Halliwell leads the opposite camp [28, 38-40]. Most in vitro antioxidant bioassays are biologically irrelevant [41]. The in vivo concentrations of biophenols are not high enough to show any antioxidant activity. In the gastrointestinal tract, biophenol concentrations are high enough that they may act as prooxidants to generate ROS in vivo [28]. Research has shown the beneficial and protective role of ROS [39]. Paradoxically, biophenols can exert cytoprotective effects by producing $a$ mild degree of oxidative stress which can induce endogenous antioxidant defence system [28]. Hence, he labels biophenols as "prooxidants" and suggests that overconsumption can down-regulate the endogenous antioxidants [38].

Biophenols Future: Multidisciplinary research teams are summoned to develop a fuller understanding of biophenol chemistry and biological activities. Progress in biophenol research is linked to advances into oxidative stress-disease relationship. Nutrigenomics, nutrigenetics, nutriproteomics, nutrapharmacology, nutraceutics and medical foods are opening new horizons in biophenol research. Phytochemical screening of novel plants for their biophenol content will continue to provide us with inexhaustible resource of structural diversity. A rigorous and systematic validation of traditional and ethno-medicines will be profitable both medically and culturally. High-throughput standardized chemical assays and ultra-performance profiling techniques are essential for drug discovery and commercialization. Application of bioinformatics to analyse the tremendous amount of data generated will increase research turn over. There is always a need to develop economic and robust in vitro bioassays that correlate with in vivo activity for biological screening. A comprehension of the chemistry behind bioassays is mandatory to correctly interpret results [24]. In antioxidant assays, structural elucidation of oxidation products and their activities 
and side reactions will allow more accurate extrapolation into biological system [38]. Search for newer molecular and cellular targets is definitely on the priority list [42].

Randomized controlled trials are vital to study the pharmacodynamics and pharmacokinetics of pure biophenols and plant extracts. Only through well designed clinical trials, we can ascertain whether biophenols in vivo act as antioxidants, prooxidants or redox modulators. Characterization of biophenol metabolites and their biological activities is an important piece in the puzzle. Experiments in healthy volunteers and patients are both required to assess the role of oxidative stress. To conduct antioxidant clinical trials, validated oxidative damage biomarkers are essential [42]. Very limited information can be found on the toxicity and adverse effects of biophenols. Intense investigation into the posology of biophenols and determination of their therapeutic indexes are required for safe and effective administration of nutraceutical and pharmaceutical preparations. Application of nanotechnology for enhanced delivery and bioavailability of biophenol dosage forms is also actively growing. Drug-drug, drug-food, drug-herb and drug-disease interactions are still largely unstudied areas that entail more attention.

Conclusion: By no means, we are witnessing a dawn of a golden era for biophenol research. The issue of the antioxidant attributes of biophenols does not lend itself to a quick and easy answer. The author has proposed the more comprehensive term "biophenols" to replace the chemically inaccurate term "polyphenols" and provided a definition for "biophenols" for the first time. More research into the biochemistry and pharmacology of biophenols in oxidative stress and oxidative damage in vivo is mandatory before we can establish whether biophenols play any significant role as antioxidants, prooxidants or redox modulators. Beyond antioxidant potential, researchers have revealed that biophenols can exert medicinal properties through enzyme modulation, receptor binding, metabolic interference, gene expression and epigenetic regulation. Currently, we stand on a peak of an iceberg. Multidisciplinary approach is essential to develop a fuller understanding of biophenol function in health and disease. Meanwhile, consumption of well-balanced biophenol-rich diet is to be encouraged to stay healthy.

Acknowledgements: I am grateful for my wife for her great support and proof-reading early drafts of this manuscript. I like to thank Associate Professor Paul Prenzler for critically reading this manuscript. Funding by Graham Centre for Agricultural Innovation and Charles Sturt University is acknowledged.

\section{REFERENCES:}

1. Barreiro-Hurle J, Gracia A, De-Magistris T, The effects of multiple health and nutrition labels on consumer food choices. Journal of Agricultural Economics. 2010, 61: 426-443.

2. Kiesel K, McCluskey JJ, Villas-Boas SB, Nutritional labeling and consumer choices. Annual Reviews of Resource Economics. 2011, 3: 141-158.

3. Heber D, An integrative view of obesity. The American Journal of Clinical Nutrition. 2010, 91: 280S-283S. 
4. Remington PL, Brownson RC, Wegner MV: Chronic disease epidemiology and control: American Public Health Association; 2010.

5. Gurib-Fakim A, Medicinal plants: traditions of yesterday and drugs of tomorrow. Molecular Aspects of Medicine. 2006, 27: 1-93.

6. Li P, Plant Natural Products in Drug Discovery. Current Organic Chemistry. 2010, 14: 1669-1669.

7. Firn RD \& Jones CG, The evolution of secondary metabolism-a unifying model. Molecular Microbiology. 2002, 37: 989-994.

8. Iwu MM \& Wootton J: Ethnomedicine and drug discovery. Vol. 1. Elsevier Science; 2002.

9. Mondello L, Nutraceuticals and separations. Analytical and Bioanalytical Chemistry. $20131-2$.

10. 1Besançon P, Debosque S, Delpeuch F: Mediterranean diet and health: current news and prospects London, UK: John Libbey Eurotext Limited; 2001.

11. Keys A, Mediterranean diet and public health: personal reflections. The American Journal of Clinical Nutrition. 1995, 61: 1321S-1323S.

12. Renaud S \& de Lorgeril M, Wine, alcohol, platelets, and the French paradox for coronary heart disease. The Lancet. 1992, 339: 1523-1526.

13. Obied HK, Prenzler PD, Omar SH, Ismael R, Servili M, Esposto S, Taticchi A, Selvaggini R, Urbani S, Pharmacology of Olive Biophenols. Advances in Molecular Toxicology. 2012, 6: 195.

14. Haslam E, Plant polyphenols: vegetable tannins revisited: CUP Archive; 1989.

15. Robards K, Prenzler PD, Tucker G, Swatsitang P, Glover W, Phenolic compounds and their role in oxidative processes in fruits. Food Chemistry. 1999, 66: 401-436.

16. Robards K, Strategies for the determination of bioactive phenols in plants, fruit and vegetables. Journal of Chromatography A. 2003, 1000: 657-691.

17. Ryan D, Robards K, Prenzler P, Antolovich M, Applications of mass spectrometry to plant phenols. Trac-Trends in Analytical Chemistry. 1999, 18: 362-372.

18. Quideau S, Deffieux D, Douat-Casassus C, Pouysegu L, Plant polyphenols: chemical properties, biological activities, and synthesis. Angewandte Chemie International Edition. 2011, 50: 586-621.

19. Romeo G \& Uccella N, Olive biophenols: taste supermolecules in Mediterranean aliment culture. Tetrahedron-Eppos. 1996, 21: 33-80.

20. Harman D, Prolongation of the normal life span by radiation protection chemicals. Journal of Gerontology. 1957, 12: 257-263.

21. Temple NJ, Antioxidants and disease: more questions than answers. Nutrition Research. 2000, 20: 449-459.

22. Harman D, The biologic clock: the mitochondria? Journal of the American Geriatrics Society. 1972, 20: 145.

23. Sies H, Oxidative stress. Academic Press Inc., Orlando, FL. 1985.

24. Obied HK, Prenzler PD, Konczak I, Rehman A-u,Robards K, Chemistry and bioactivity of olive biophenols in some antioxidant and antiproliferative in vitro bioassays. Chemical Research in Toxicology. 2008, 22: 227-234.

25. Leopoldini M, Russo N,Toscano M, The molecular basis of working mechanism of natural polyphenolic antioxidants. Food Chemistry. 2011, 125: 288-306. 
26. Nijveldt RJ, van Nood E, van Hoorn DEC, Boelens PG, van Norren K,van Leeuwen PAM, Flavonoids: a review of probable mechanisms of action and potential applications. American Journal of Clinical Nutrition. 2001, 74: 418-425.

27. Prochazkova D, Bousova I, Wilhelmova N, Antioxidant and prooxidant properties of flavonoids. Fitoterapia. 2011, 82: 513-523.

28. Halliwell B, Are polyphenols antioxidants or pro-oxidants? What do we learn from cell culture and in vivo studies? Archives of Biochemistry and Biophysics. 2008, 476: 107.

29. Tucker G \& Robards K, Bioactivity and structure of biophenols as mediators of chronic diseases. Critical Reviews in Food Science and Nutrition. 2008, 48: 929-966.

30. Almoosawi S, Tsang C, Ostertag LM, Fyfe L, Al-Dujaili EAS, Differential effect of polyphenol-rich dark chocolate on biomarkers of glucose metabolism and cardiovascular risk factors in healthy, overweight and obese subjects: a randomized clinical trial. Food \& Function. 2012, 3: 1035-1043.

31. Pase MP, Scholey AB, Pipingas A, Kras M, Nolidin K, Gibbs A, Wesnes K, Stough $\mathrm{C}$, Cocoa polyphenols enhance positive mood states but not cognitive performance: a randomized, placebo-controlled trial. Journal of Psychopharmacology. 2013, 27: 4518.

32. Joseph JA, Shukitt-Hale B, Lau FC, Fruit polyphenols and their effects on neuronal signaling and behavior in senescence, in Biogerontology: Mechanisms and Interventions, S.I.S. Rattan and S. Akman, Editors. 2007, Blackwell Publishing: Oxford. p. 470-485.

33. Wu KJ, Hsieh MT, Wu CR, Wood WG,Chen YF, Green tea extract ameliorates learning and memory deficits in ischemic rats via its active component polyphenol epigallocatechin-3-gallate by modulation of oxidative stress and neuroinflammation. Evidence-Based Complementary and Alternative Medicine. 2012. Article ID 163106

34. Choi DY, Lee YJ, Hong JT,Lee HJ, Antioxidant properties of natural polyphenols and their therapeutic potentials for Alzheimer's disease. Brain Research Bulletin. 2012, 87: 144-153.

35. Schaffer S, Asseburg H, Kuntz S, Muller WE, Eckert GP, Effects of polyphenols on brain ageing and alzheimer's disease: focus on mitochondria. Molecular Neurobiology. 2012, 46: 161-178.

36. Obied HK, Allen MS, Bedgood DR, Prenzler PD, Robards K, Stockmann R, Bioactivity and analysis of biophenols recovered from olive mill waste. Journal of Agricultural and Food Chemistry. 2005, 53: 823-837.

37. Visioli F, Hagen TM, Nutritional strategies for healthy cardiovascular aging: focus on micronutrients. Pharmacological Research. 2007, 55: 199-206.

38. Halliwell B, Free radicals and antioxidants-quo vadis? Trends in pharmacological sciences. 2011, 32: 125-130.

39. Halliwell B, The antioxidant paradox: less paradoxical now? British Journal of Clinical Pharmacology. 2012.

40. Halliwell B, Rafter J,Jenner A, Health promotion by flavonoids, tocopherols, tocotrienols, and other phenols: direct or indirect effects? Antioxidant or not? The American Journal of Clinical Nutrition. 2005, 81: 268S-276S.

41. Bartosz G, Total antioxidant capacity. Advances in Clinical Chemistry. 2003, 37: 219-292. 
42. Visioli F, Lastra CADL, Andres-Lacueva C, Aviram M, Calhau C, Cassano A, D'Archivio M, Faria A, Favé G,Fogliano V, Polyphenols and human health: a prospectus. Critical Reviews in Food Science and Nutrition. 2011, 51: 524-546. 